Apidologie, 1977, 8 (4), 419-426.

\title{
ZUR KENNTNIS DER ZUSAMMENSETZUNG DES HONIGTAUES
}

\author{
Étude de la composition du miellat \\ Hubert ZIEGLER und Sigrid PENTH \\ Institut für Botanik und Mikrobiologie der \\ Technischen Universität München \\ München (B.R.D.)
}

\section{SUMMARY \\ CONTRIBUTION TO THE KNOWLEDGE OF HONEYDEW-COMPOSITION}

Honeydew produced by several aphid species and one coccid was analyzed by chemical and microbiological methods. In any of the honeydew samples the water-soluble vitamins ascorbic acid, thiamine, biotin, folic acid, pyridoxine, riboflavin, niacin, pantothenic acid, and myo-inositol were identified. All analyzed samples contained ATP, ADP and AMP; the energy charge was between 0,13 and 0,63 , which is considerably below the values determined in the sieve tube exudates.

\section{ZUSAMMENFASSUNG}

Honigtau von verschiedenen Aphidenarten und einer Coccide wurde mit chemischen und mokrobiologischen Methoden analysiert. In allen geprüften Proben konnten die wasserlöslichen Vitamine Ascorbinsäure, Thiamin, Biotin, Folsäure, Pyridoxin, Riboflavin, Niacin, Pantothensäure und myo-Inosit nachgewiesen werden. Alle darauf analysierten HonigtauProben enthielten ATP, ADP und AMP; die " energy charge " lag zwischen 0,13 und 0,63 und war damit wesentlich niedriger als in den bisher untersuchten Siebröhrensäften.

\section{I. - EINLEITUNG}

Honigtau wie Nektar leiten sich letztlich vom Inhalt der Assimilatleit* bahnen der Pflanzen ab, wobei dieser im einen Fall durch die (z.T. spezifische) 
Entnahme von Stoffen durch die Siebröhren-ausbeutenden Insekten, im anderen Fall durch das Zurückhalten (bzw. die Rückresorption) einzelner Stoffe durch das Nektarium verändert ist. Über die Zusammensetzung des Honigtaues gibt es zahlreiche Arbeiten, wobei u.a. Kohlenhydrate, Zuckeralkohole, organische Säuren, Stickstoffsubstanzen, Mineralstoffe, Wuchsstoffe und einige Enzyme analysiert wurden (vgl. Übersicht bei Kloft, Maurizio u. Kaeser, 1965; Klof T, 1968). Hier soll über den Gehalt einiger Honigtausorten an wasserlöslichen Vitaminen und an Komponenten des Adenylsäuresystems berichtet werden, die bisher unseres Wissens noch nicht getestet worden waren.

\section{II. - MATERIAL UND METHODE}

Die Pflanzen mit den auf ihnen saugenden Insekten stammten aus dem Botanischen Garten und den Gewächshäusern des Botanischen Institutes der TH Darmstadt. Die Bestimmung der Aphiden und der Coccide übernahmen dankenswerterweise die Herren Prof. Dr. K. Heinze, Berlin, und Prof. Dr. H. Schmutterer, Gießen.

Die mit Honigtau-produzierenden Insekten befallenen Pflanzen oder Pflanzenteile (diese in Leitungswasser gestellt) wurden in einem verdunkelten Raum bei 19-20 ${ }^{\circ} \mathrm{C}$ gehalten, der Honigtau auf Glasplatten aufgefangen, mit aqua dest. aufgenommen, gefriergetrocknet und bis zur Analyse bei -30 oC aufbewahrt.

Die Vitamine wurden chemisch (Ascorbinsäure) oder mikrobiologiseh (alle anderen), wie in Ziegler u. Ziegler (1962) angegeben, bestimmt. Da nach Buchner (1966) Honigtauhonig gegenüber Bakterien wachstumshemmend wirken kann, wurde geprüft, ob er auch gegenüber den zum Vitamintest verwendeten Bakterien-und Pilzstämmen ähnliche Wirkungen hat. Dies war aber sowenig der Fall wie bei einem von R. BuchNeR zur Verfügung gestellten Honigtau-Honig (Tanne) und einem Blütenhonig (Robinie).

ATP wurde fluorimetrisch mit dem Firefly-Enzymtest, ADP und AMP mit Hilfe der Enzymtest-Kombination TC-K (Fa. Boehringer und Söhne, Mannheim) bestimmt (vgl. KLUGE 1. ZiEgler, 1964). Zum ATP-Test wurden 3 und $6 \mathrm{mg}$ Honigtau-Trockensubstanz in aqua bidest. gelöst, während für den ADP- und AMP-Nachweis verschiedene Mengen, je nach Honigtauart, gewählt wurden.

\section{III. - ERGEBNISSE UND DISKUSSION}

\section{1. - Vitaminbestimmungen}

Wie aus Tabelle 1 hervorgeht, sind alle geprüften Vitamine in den verschiedenen Honigtausorten nachweisbar. Die Konzentration der einzelnen Vitamine ist erwartungsgemäß bei den verschiedenen Honigtauproben unterschiedlich, auch bei Herkunft von der gleichen Aphidenart (z.B. Aphis fabae) auf verschiedenen Wirtspflanzen, von der gleichen Wirtspflanze (Urtica dioica) mit verschiedenen Aphiden, oder bei gleicher Wirtspflanze und gleichem Insekt zu verschiedenen Jahreszeiten. Soweit Vergleiche möglich sind, sind auffallende Unterschiede in der Konzentration dieser wasserlöslichen Vitamine im Honigtau einerseits und in den Siebröhrensäften (Ziegler u. Ziegler, 1962) 
TA8. 1. - Vitamingehalte einiger Honigtausorten. Die Mengenangaben bei den einzelnen Vitaminen bedeuten zuerst $\mu \mathrm{g} / \mathrm{mg}$ Gefriertrockensubstanz und dann $\mathrm{mg} / \mathrm{g}$ Zucker.

'1ABL. 1. - Teneur en vitamines de quelques types de miellat. Les quantités de vitamines sont exprimées d'abord en $\mu \mathrm{m} / \mathrm{mg}$ de substance sèche congelée, puis en $\mathrm{mg} / \mathrm{g}$ de suere.

\begin{tabular}{|c|c|c|c|c|}
\hline $\begin{array}{c}\text { Wirtspflanze } \\
\text { Plante-hôte } \\
\text { (Insekt) } \\
\text { (Insecte) }\end{array}$ & $\begin{array}{l}\text { Sammel- } \\
\text { zeit } \\
\text { Mois }\end{array}$ & $\begin{array}{l}\text { Ascorbin- } \\
\text { säure } \\
\text { acide ascorbique }\end{array}$ & $\begin{array}{l}\text { Thiamin } \\
\text { thiamine }\end{array}$ & $\begin{array}{l}\text { Biotin } \\
\text { biotine }\end{array}$ \\
\hline \multicolumn{5}{|l|}{$\begin{array}{l}\text { (Blattläuse) } \\
\text { (Pucerons) }\end{array}$} \\
\hline $\begin{array}{l}\text { Urtica dioica } \mathrm{L} . \\
\text { (Aphis urticata } \mathrm{F} .)\end{array}$ & Sept. & $1,05 / 1,5$ & $2,7 \cdot 10-5 / 3,7 \cdot 10-^{12}$ & $6,3 \cdot 10-^{12} / 8 \cdot 10-^{12}$ \\
\hline $\begin{array}{l}\text { Euonymus hamiltonianus } \\
\text { Wall. (Aphis fabae Scop.) }\end{array}$ & Mai & $0,74 / 4,8$ & $2 \cdot 10-5 / 13 \cdot 10-^{5}$ & $9 \cdot 10-^{12} / 58 \cdot 10-^{12}$ \\
\hline $\begin{array}{l}\text { Vicia faba } \mathbf{L} . \\
\text { (Aphis fabae Scop.) }\end{array}$ & Mai & $0,32 / 2,8$ & - & $15 \cdot 10-12 / 138 \cdot 10-^{12}$ \\
\hline $\begin{array}{l}\text { Sambucus nigra L. } \\
\text { (Aphis sambuci L.) }\end{array}$ & Juni & $0,76 / 1,4$ & $2,7 \cdot 10-5 / 5 \cdot 10-^{5}$ & $4 \cdot 10-12 / 74 \cdot 10-12$ \\
\hline $\begin{array}{l}\text { Hibiscus rosa-sinensis L. } \\
\text { (Myzus ornatus Laing. } \\
\text { u. Myzodes persicae Sulz) }\end{array}$ & $\begin{array}{l}\text { Dez. } \\
\text { März }\end{array}$ & $\begin{array}{l}0,25 /- \\
0,4,2 /-\end{array}$ & - & $\begin{array}{l}6,5 \cdot 10-12 /- \\
7,2 \cdot 10-12 /-\end{array}$ \\
\hline $\begin{array}{l}\text { Clematis dioscoreifolia } \\
\text { Levl. et Van. } \\
\text { (Myzodes varians Davids.) }\end{array}$ & Nov. & $1,5 / 4,5$ & $2,6 \cdot 10-^{5} / 7,8 \cdot 10-^{5}$ & - \\
\hline $\begin{array}{l}\text { Cornus sanguinea } \mathrm{L} . \\
\text { (Anoecia corni F.) }\end{array}$ & $\begin{array}{l}\text { Okt. } \\
\text { Mai }\end{array}$ & $\begin{array}{l}5,7 / 9 \\
4,6 / 7\end{array}$ & $4,7 \cdot 10-5 / 7,4 \cdot 10-^{5}$ & $\begin{array}{l}9,7 \cdot 10-_{12}^{12} / 15 \cdot 10-_{12} \\
7,7 \cdot 10-^{12} / 12 \cdot 10-^{12}\end{array}$ \\
\hline $\begin{array}{l}\text { Urtica dioica L. } \\
\text { (Microlophium evansi } \\
\text { Theob.) }\end{array}$ & Juni & $0,31 / 1,5$ & - & $9,8 \cdot 10-12 / 47 \cdot 10-12$ \\
\hline $\begin{array}{l}\text { Prunus padus L. } \\
\text { (Rhopalosiphum padi L.) }\end{array}$ & $\begin{array}{l}\text { Okt. } \\
\text { Mai }\end{array}$ & $\begin{array}{l}5,3 / 15,8 \\
0,3 / 0,9\end{array}$ & $6 \cdot 10-5 / 20 \cdot 10-^{5}$ & $\begin{array}{c}6 \cdot 10-^{12} / 20 \cdot 10-^{12} \\
10 \cdot 10-^{12} / 35 \cdot 10-^{12}\end{array}$ \\
\hline $\begin{array}{l}\text { Prunus domestica L. } \\
\text { (Hyalopterus pruni } \\
\text { Geoffr.) }\end{array}$ & Mai & $0,35 / 0,8$ & $8,6 \cdot 10-6 / 19,5 \cdot 10-^{5}$ & $4,5 \cdot 10-12 / 10 \cdot 10-^{12}$ \\
\hline
\end{tabular}

(Schildlaus)

(Cochenilles)

\begin{tabular}{l|l|l|l|l}
\hline $\begin{array}{l}\text { Ficus benjaminii L. } \\
\text { (Coccus hesperidum L.) }\end{array}$ & Jan. & $2,3 / 3,5$ & $1 \cdot 10-4 / 16,4 \cdot 10-^{5}$ & $3,8 \cdot 10-12 / 5,7 \cdot 10-12$ \\
\hline
\end{tabular}




\begin{tabular}{c|c|c|c|c}
$\begin{array}{c}\text { Wirtspflanze } \\
\text { Plante-hôte } \\
\vdots\end{array}$ & $\begin{array}{c}\text { Sammel- } \\
\text { zeit } \\
\text { (Insekt) } \\
\text { (Insecte) }\end{array}$ & $\begin{array}{c}\text { Folsäure } \\
\text { Acide folique }\end{array}$ & $\begin{array}{c}\text { Pyridoxin } \\
\text { Pyridoxine }\end{array}$ & $\begin{array}{c}\text { Riboflavin } \\
\text { Riboflavine }\end{array}$ \\
\hline
\end{tabular}

(Blattläuse)

(Pucerons)

\begin{tabular}{|c|c|c|c|c|}
\hline $\begin{array}{l}\text { Urtica dioica } \mathrm{L} . \\
\text { (Aphis urticata } \mathrm{F} .)\end{array}$ & Sept. & $4 \cdot 10-5 / 5 \cdot 10-^{5}$ & - & - \\
\hline $\begin{array}{l}\text { Euonymus hamiltonianus } \\
\text { Wall. (Aphis fabae Scop.) }\end{array}$ & Mai & $1 \cdot 10-4 / 6,5 \cdot 10^{4}$ & $4 \cdot 10^{4} / 27 \cdot 10^{4}$ & $1 \cdot 10-4 / 7 \cdot 10-4$ \\
\hline $\begin{array}{l}\text { Vicia faba L. } \\
\text { (Aphis fabae Scop.) }\end{array}$ & Mai & $1,7 \cdot 10-4 / 15 \cdot 10-^{4}$ & $1,8 \cdot 10-4 / 16 \cdot 10-^{4}$ & $1 \cdot 10-4 / 12 \cdot 10-4$ \\
\hline $\begin{array}{l}\text { Sambucus nigra } L . \\
\text { (Aphis sambuci L.) }\end{array}$ & Juni & $1,4 \cdot 10-4 / 2,6 \cdot 10-4$ & $4,7 \cdot 10-4 / 8,8 \cdot 10-^{4}$ & $0,7 \cdot 10-4 / 1,3 \cdot 10-4$ \\
\hline $\begin{array}{l}\text { Hibiscus rosa-sinensis L. } \\
\text { (Myzus ornatus Laing. } \\
\text { u. Myzodes persicae Sulz). }\end{array}$ & $\begin{array}{l}\text { Dez. } \\
\text { März }\end{array}$ & $\begin{array}{l}5 \cdot 10 \cdot 5 /- \\
2 \cdot 10 \cdots 5\end{array}$ & - & - \\
\hline $\begin{array}{l}\text { Clematis dioscoreifolia } \\
\text { Levl. et Van. } \\
\text { (Myzodes varians Davids.) }\end{array}$ & Nov. & - & $16,6 \cdot 10-4 / 49 \cdot 10-4$ & $2 \cdot 10-4 / 5,9 \cdot 10_{-4}^{-4}$ \\
\hline $\begin{array}{l}\text { Cornus sanguinea } \mathrm{L} . \\
\text { (Anoecia corni F.) }\end{array}$ & $\begin{array}{l}\text { Okt. } \\
\text { Mai }\end{array}$ & $\begin{array}{l}1 \cdot 10-4 / 1,6 \cdot 10-4 \\
7 \cdot 10-4 / 12 \cdot 10-4\end{array}$ & - & $\begin{array}{c}0,8 \cdot 10-4 / 1,3 \cdot 10-4 \\
-\end{array}$ \\
\hline $\begin{array}{l}\text { Urtica dioica L. } \\
\text { (Microlophium evansi } \\
\text { Theob.) }\end{array}$ & Juni & $3 \cdot 10^{-4} / 15 \cdot 10^{4}$ & $3 \cdot 10-1 / 15 \cdot 10-4$ & $0,5 \cdot 10-4 / 2,4 \cdot 10-4$ \\
\hline $\begin{array}{l}\text { Prunus padus L. } \\
\text { (Rhopalosiphum padi L.) }\end{array}$ & $\begin{array}{l}\text { Okt. } \\
\text { Mai }\end{array}$ & $\begin{array}{l}2 \cdot 10 \omega^{5} / 6 \cdot 10^{5} \\
2 \cdot 10-4 / 8 \cdot 10-^{4}\end{array}$ & - & - \\
\hline $\begin{array}{l}\text { Prunus domestica L. } \\
\text { (Hyalopterus pruni } \\
\text { Geoffr.) }\end{array}$ & Mai & $1,6 \cdot 10-4 / 3,7 \cdot 10-4$ & $1,8 \cdot 10-4 / 4 \cdot 10-4$ & $0,9 \cdot 10-4 / 2 \cdot 10-4$ \\
\hline
\end{tabular}

(Schildlaus)

(Cochenilles)

\begin{tabular}{l|c|c|c|c}
\hline $\begin{array}{l}\text { Ficus benjaminii L. } \\
\text { (Coccus hesperidum L.) }\end{array}$ & Jan. & $2 \cdot 10-5 / 3 \cdot 10-5$ & - & - \\
\hline
\end{tabular}




\begin{tabular}{|c|c|c|c|c|}
\hline $\begin{array}{c}\text { Wirtspflanze } \\
\text { Plante-hôte } \\
\text { (Insekt) } \\
\text { (Insecte) }\end{array}$ & $\begin{array}{c}\text { Sammel- } \\
\text { zeit } \\
\text { Mois }\end{array}$ & $\begin{array}{l}\text { Niacin } \\
\text { Niacine }\end{array}$ & $\begin{array}{l}\text { Pantothensäure } \\
\text { Acide pentothénique }\end{array}$ & $\begin{array}{l}\text { myo-Inosit } \\
\text { Myo-inositol }\end{array}$ \\
\hline
\end{tabular}

\section{(Blattläuse)}

(Pucerons)

\begin{tabular}{|c|c|c|c|c|}
\hline $\begin{array}{l}\text { Urtica dioica } \mathrm{L} . \\
\text { (Aphis urticata } \mathrm{F} .)\end{array}$ & Sept. & $4,6 \cdot 10^{-3} / 6,5 \cdot 10^{-9}$ & $2,2 \cdot 10^{-3} / 3,4 \cdot 10^{-3}$ & $38,6 / 60 \cdot 8$ \\
\hline $\begin{array}{l}\text { Euonymus hamiltonianus } \\
\text { Wall. (Aphis fabae Scop.) }\end{array}$ & Mai & $0,2 / 1,5$ & $12 \cdot 10^{-3} / 0,7$ & $20,4 / 133$ \\
\hline $\begin{array}{l}\text { Vicia faba } \mathrm{L} . \\
\text { (Aphis fabae Scop.) }\end{array}$ & Mai & $0,2 / 1,77$ & $7 \cdot 10^{-3} / 0,6$ & $1,4 / 12,3$ \\
\hline $\begin{array}{l}\text { Sambucus nigra } \mathrm{L} \text {. } \\
\text { (Aphis sambuci L.) }\end{array}$ & Juni & $6 \cdot 10^{-3} / 0,1$ & $1 \cdot 10^{-3} / 2,2 \cdot 10^{-3}$ & $16,5 / 30,7$ \\
\hline $\begin{array}{l}\text { Hibiscus rosa-sinensis } \mathrm{L} . \\
\text { (Myzus ornatus Laing. } \\
\text { u. Myzodes persicae Sulz.) }\end{array}$ & $\begin{array}{l}\text { Dez. } \\
\text { März }\end{array}$ & $4,8 \cdot 10^{-3} /-$ & $\begin{array}{l}4,9 \cdot 10^{-3} /= \\
5,7 \cdot 10^{-3} /-\end{array}$ & $\overline{1,0 /}$ \\
\hline $\begin{array}{l}\text { Clematis dioscoreifolia } \\
\text { Levl. et Van. } \\
\text { (Myzodes varians Davids.) }\end{array}$ & Nov. & - & - & $7,58 / 22,3$ \\
\hline $\begin{array}{l}\text { Cornus sanguinea } \mathrm{L} \text {. } \\
\text { (Anocia corni } \mathrm{F} .)\end{array}$ & $\begin{array}{l}\text { Okt. } \\
\text { Mai }\end{array}$ & $\begin{array}{l}8,7 \cdot 10^{-3} / 0,14 \\
8,2 \cdot 10^{-3} / 0,13\end{array}$ & $\begin{array}{l}1,3 \cdot 10^{-3} / 2,0 \cdot 10^{-3} \\
3,2 \cdot 10^{-3} / 4,9 \cdot 10^{-3}\end{array}$ & $\begin{array}{l}38,6 / 60,8 \\
33,2 / 50,6\end{array}$ \\
\hline $\begin{array}{l}\text { Urtica dioica L. } \\
\text { (Microlophium evansi } \\
\text { Theob.) }\end{array}$ & Juni & $9,2 \cdot 10^{-3} / 0,44$ & $3 \cdot 10^{-3} / 14,5 \quad 10^{-3}$ & $8,78 / 42,4$ \\
\hline $\begin{array}{l}\text { Prunus padus L. } \\
\text { (Rhopalosiphum padi L.) }\end{array}$ & $\begin{array}{l}\text { Okt. } \\
\text { Mai }\end{array}$ & $\begin{array}{c}5 \cdot 10^{-3} / 0,15 \\
5,4 \cdot 10^{-3} / 0,18\end{array}$ & $\begin{array}{l}3,8 \cdot 10^{-3} / 11,3 \cdot 10^{-3} \\
6,3 \cdot 10^{-3} / 21,3 \cdot 10^{-3}\end{array}$ & $\begin{array}{l}12,9 / 38,4 \\
10,7 / 36,6\end{array}$ \\
\hline $\begin{array}{l}\text { Prunus domestica L. } \\
\text { (Hyalopterus pruni } \\
\text { Geoffr.) }\end{array}$ & Mai & $4,8 \cdot 10^{-3} / 0,11$ & $4,6 \cdot 10^{-3} / 10,1 \cdot 10^{-3}$ & $12,1 / 27,3$ \\
\hline
\end{tabular}

(Schildlaus)

(Cochenilles)

\begin{tabular}{l|c|c|c|c}
\hline $\begin{array}{l}\text { Ficus benjaminii L. } \\
\text { (Coccus hesperidum L.) }\end{array}$ & Jan. & $2,8 \cdot 10,3 / 4,4 \cdot 10^{-3}$ & $2 \cdot 10^{-3} / 3 \cdot 10^{-3}$ & $2,62 / 3,9$ \\
\hline
\end{tabular}


TAB. 2. - Gehalt an ATP, ADP und AMP ( $\mu$ g/mg Tr.-Gew.) sowie " energy charge » in verschiedenen Honigtausorten. (NB : Bei der Ermittlung der « energy charge » gehen die Molaritaten in die Berechnung ein!)

TABL. 2, - Teneur en ATP, en $A D P$ et en $A M P(\mu \mathrm{g} / \mathrm{mg}$ de poids sec) et charge énergétique de divers types de miellat. (NB : Pour calculer la charge énergétique, on tient compte des molarités!)

\begin{tabular}{|c|c|c|c|c|c|}
\hline $\begin{array}{c}\text { Wirtspflanze } \\
\text { Plante-hôte } \\
\text { (Insekt) } \\
\text { (Insecte) }\end{array}$ & $\begin{array}{l}\text { Sammelzeit } \\
\text { Mois }\end{array}$ & $\begin{array}{l}\text { ATP } \\
\text { ATP }\end{array}$ & $\begin{array}{l}\text { ADP } \\
\text { ADP }\end{array}$ & $\begin{array}{l}\text { AMP } \\
\text { AMP }\end{array}$ & $\begin{array}{c}\text { energy } \\
\text { charge } \\
\text { Charge } \\
\text { énergétique }\end{array}$ \\
\hline \multicolumn{6}{|l|}{$\begin{array}{c}\text { (Blattläuse) } \\
\text { (Pucerons) }\end{array}$} \\
\hline $\begin{array}{l}\text { Euonymus hamiltonianus } \\
\text { (Aphis fabae) }\end{array}$ & Mai & 0,61 & 1,5 & 1,12 & 0,37 \\
\hline $\begin{array}{l}\text { Vicia faba } \\
\text { (Aphis fabae) }\end{array}$ & Mai & 0,28 & 2,31 & 1,74 & 0,28 \\
\hline $\begin{array}{l}\text { Sambucus nigra } \\
\text { (Aphis sambuci) }\end{array}$ & Juni & 0,48 & 0,47 & 0,37 & 0,48 \\
\hline $\begin{array}{l}\text { Clematis dioscoreifolia } \\
\text { (Myzodes varians) }\end{array}$ & Nov. & 0,26 & 2,6 & 1,51 & 0,32 \\
\hline $\begin{array}{l}\text { Cornus sanguinea } \\
\text { (Anæecia corni) }\end{array}$ & $\begin{array}{l}\text { Okt. } \\
\text { Mai }\end{array}$ & $\begin{array}{l}0,62 \\
0,32\end{array}$ & $\underline{0,51}$ & $\underline{0,69}$ & $\underline{0,41}$ \\
\hline $\begin{array}{l}\text { Urtica dioica } \\
\text { (Microlophium evansi) }\end{array}$ & Juni & 0,49 & - & - & - \\
\hline $\begin{array}{l}\text { Prunus padus } \\
\text { (Rhopalosiphum padi) }\end{array}$ & $\begin{array}{l}\text { Okt. } \\
\text { Mai }\end{array}$ & $\begin{array}{l}0,3 \\
0,05\end{array}$ & $\begin{array}{l}1,06 \\
\mathbf{0 , 3 5}\end{array}$ & $\begin{array}{l}0,92 \\
0,97\end{array}$ & $\begin{array}{l}0,32 \\
0,13\end{array}$ \\
\hline $\begin{array}{l}\text { Prunus domestica } \\
\text { (Hyalopterus pruni) }\end{array}$ & Mai & 0,13 & 0,9 & 1,09 & 0,23 \\
\hline
\end{tabular}

(Schildlaus)

(Cochenilles)

\begin{tabular}{l|l|l|l|l|l|l}
\hline $\begin{array}{l}\text { Ficus benjaminii } \\
\text { (Coccus hesperidum) }\end{array}$ & Jan. & 1,66 & 0,8 & 0,52 & 0,63 \\
\hline
\end{tabular}


andererseits nicht festzustellen; d.h., die phloemausbeutenden Insekten nehmen die genannten, für sie fast durchwegs lebensnotwendigen (vgl. Auclarr, 1965: Dadd, Krieger u. Mittler, 1967) Vitamine etwa in gleichem Maße auf wie den Zucker, sodaß sich der Vitamingehalt bei Bezug auf den Zucker nicht wesentlich ändert. Vielleicht bildet die einzige untersuchte Coccide eine Ausnahme : Sie hat allgemein einen relativ geringen Vitamingehalt im Honigtau, und es könnte sein, daß sie einen größeren Vitaminbedarf (geringere Eigenversorgung über Symbionten ?) hat als die Aphiden. Dies müßte aber durch eingehendere Untersuchungen erhärtet werden. Auch gegenüber dem Vitaruingehalt des Nektars (Ziegler, LüTtge u. LütTge, 1964) ist der des Honigtaues nicht sehr verschieden. Die Bienen werden ihren Bedarf aber wohl vorwiegend durch den Pollen decken, der besonders vitaniureich ist (vgl. Literatur bei Ziegler, Lüttge u. LüTtge).

\section{2. - Bestimmungen von $A T P, A D P$ und $A M P$}

Sämtliche geprüften Honigtausorten enthalten alle Glieder des Adenylsäuresystems (Tab. 2). Obwohl ATP offensichtlich für phloemsaugende Insekten förderlich ist (HerteL, 1974), wird es sowenig wie die Vitamine vollständig aus der Nahrung resorbiert. Allerdings ist im Honigtau das Adenylsäuresystem gegenüber dem Siebröhrensaft (KLUGE u. ZiEGLER, 1964) zum energieärmeren Zustand hin verschoben. Dies kommt in der « energy charge » zum Ausdruck:

$$
1 / 2 \frac{[\mathrm{ADP}]+2[\mathrm{ATP}]}{[\mathrm{AMP}]+[\mathrm{ADP}]+[\mathrm{ATP}]} \text {. }
$$

Sie kann grundsätzlich zwischen 1,0 (nur ATP vorhanden) und 0 (nur AMP) variieren. Die energy charge im Siebröhrensaft von Robinia betrug 0,88 , von Tilia 0,77 (vgl. ZIEGLER), während sie in den geprüften Honigtausorten der Aphiden zwischen 0,13 und 0,48, bei der Coccide bei 0,63 lag. Nimmt man an, daß die energy charge im Siebröhrensaft der in dieser Arbeit untersuchten Arten ähnlich liegt wie bei den beiden Bäumen, so würde dies darauf hinweisen, daß die Insekten (vor allem die Aphiden) bevorzugt die energiereicheren Glieder des Adenylsäuresystems absorbieren (oder daß die energiereichen Bindungen im ATP und ADP bei der Passage durch die Insekten teilweise gespalten werden).

Eingegangen im März 1977.

Reçu pour publication en mars 1977.

\section{RÉSUMÉ}

On a analysé le miellat de différentes espèces d'Aphidiens et de quelques Coccidés par des méthodes chimiques et microbiologiques. Dans tous les échantillons testés on a pu mettre en évidence les vitamines hydrosolubles suivantes : acide ascorbique, thiamine, biotine, acide 
folique, pyridoxine, riboflavine, niacine, acide pentothénique et méso-inositol. Tous les échantillons analysés renfermaient de l'ATP, de l'ADP et de l'AMP; la charge énergétique se situait entre 0,13 et 0,63 , ce qui est nettement inférieur aux valeurs déterminées pour les exsudats des tubes criblés.

\section{LITERATUR}

Auclair J. L., 1965. - Feeding and nutrition of the pea aphid Acyrthosiphon pisi (Homoptera : Aphididae) on chemically defined diets of various $\mathrm{pH}$ and nutrial levels. Ann. Ent. Soc. Amer., 58, 855-875.

Buchner R., 1966. - Vergleichende Untersuchungen über die antibakterielle Wirkung von Blüten- und Honigtanhonigen. S. W. dtsch. Imker, 18, 240-24.1.

Dadd R. H., Kriegen D. L., Mittler, T. E., 1967. - Studies on the artificial feeding of the aphid Myzus persicae (SULzer)-IV. Requirements for water-soluble vitamins and ascorbic acid. J. Inseet Physiol., 13, 249-272.

Hertel R., 1974. - Einfluß von ATP in einer holidischen Diät auf Myzus persicae (Sulz.) (Aphidina). Experientia, 30, 775-776.

KLOFr W., 1968. - Les insectes producteurs de miellat. In : Traité de Biologie de l'Abeille. Ed. R. Chauvin, S. 248-262. Paris : Masson et Cie.

Kloft W., Maurizio A., Kaeser W., 1965. - Das Waldhonigbuch. München : EhrenwirthVerlag.

Kluge M., Ziegler H., 1964. - Der ATP-Gehalt der Siebröhrensäfte von Laubbäumen. Planta 61, 167-177.

Ziegler H., 1976. - Nature of transported substances. Transport in Plants I, Encyclopedia Plant Physiology, New Series, Vol. 1, 59-100. Berlin-Heidelberg-New York : SpringerVerlag.

Ziegler H., Lüttge U., LütTge U., 1964. - Die wasserlöslichen Vitamine des Nektars. Flora, 154, 215-229.

Ziegler H., Zieglen I., 1962. - Die wasserlöslichen Vitamine in den Siebröhrensäften einiger Bäume. Flora, 152, 257-287. 